\title{
„No bees, no Bembel!“ - oder: Was das „Stöffche“ mit Bestäubung zu tun hat
}

\author{
Katja Heubach \& Torsten Collet
}

\begin{abstract}
Cider is the traditional drink of Frankfurt. After phylloxera destroyed the local grapevines by the end of the 19th century, more orchards for cider production were created. To fruit, blossoms need to be pollinated by wild and honey bees as well as other insects. They therefore provide an important ecosystem service. Without those pollinators, the apple harvest would be low in quantity and quality. The Frankfurt cider jug (Bembel) would remain empty: No bees, No Bembel! The aspect of pollination, which is also so important for humans, is highlighted in a variety of activities in the Palmengarten and Botanical Garden Frankfurt as part of the lead topic „flower and pollinator biology“. As a central element of this lead topic, the new Flower and Butterfly House, opening in 2021, displays free-flying tropical butterflies and a permanent exhibition on plant and insect interaction: „Dusted off? - flowers and their pollinators“. In the outdoors of the Palmengarten and the Botanical Garden, a large variety of wild insects can be observed, so to consolidate theoretical knowledge with practical experience.
\end{abstract}

\section{Zusammenfassung}

Apfelwein, das „Stöffche“, gilt als Frankfurter Nationalgetränk. Nach Zerstörung der lokalen Weinstöcke durch die Reblaus am Ende des 19. Jahrhunderts wurden vermehrt Streuobstwiesen für die Apfel(wein)produktion angelegt. Früchte können nur geerntet werden, wenn Insekten zuvor die Apfelblüten bestäubt haben. Honig- und Wildbienen sowie andere Insekten erbringen für uns deshalb eine wichtige Ökosystemleistung. Ohne die Bestäuber gäbe es keine Äpfel, keinen Apfelwein und bliebe der Frankfurter Apfelweinkrug (Bembel) leer: „No bees, no Bembel!“. Der auch für uns Menschen so wichtige Aspekt der Bestäubung wird im Palmengarten und Botanischen Garten Frankfurt im Rahmen des Leitthemas „Blüten- und Bestäuberbiologie“vielseitig beleuchtet. Herzstück des Leitthemas ist das im Jahr 2021 eröffnete neue Blüten- und Schmetterlingshaus mit frei fliegenden tropischen Schmetterlingen sowie die zugehörige Ausstellung „Abgestaubt! - Von Blüten und ihren Bestäubern“. Im Freiland von Palmengarten und Botanischen Garten können die Besucher*innen eine Vielzahl wildlebender Insektenarten beobachten und so theoretisches Wissen mit praktischem Erleben festigen.

\section{Das Stöffche: eine kurze Kulturbeschreibung}

Klingt es Ihnen auch in den Ohren, wenn von „Frau Rauscher aus der Klappergass““ die Rede ist? Und gehören Merkmalsbeschreibungen wie „sauer gespritzt“, „süß gespritzt“ oder sogar „tief gespritzt" zu Ihrem Alltags-Wortschatz? Wie steht es um Ihr Wissen zur Bedeutung des Fichtenkranzes in Frankfurt? Gut? - Dann sind Sie mit Sicherheit ein wahrer Verfechter, eine wahre Liebhaberin des Frankfurter Nationalgetränks.

Denn schon Frau Rauscher wusste: Ein Leben ohne „Ebbelwoi“ ist einfach keins! So denkt denn auch die/der gemeine Frankfurter*in, und auch die Zugereisten sind - wenngleich mitunter mit Verzögerung - meist sehr angetan vom herben Gesöff, das ehemals überwiegend „dribbdebach“ (hessisch für „drüben vom Bach“, also auf der südlichen Mainseite, in Sachsenhausen) ausgeschenkt wurde. Heute wird auch auf der anderen Seite des Baches und darüber hinaus im ganzen Land fleißig Ebbelwoi getrunken: Der heutige Durchschnitts-

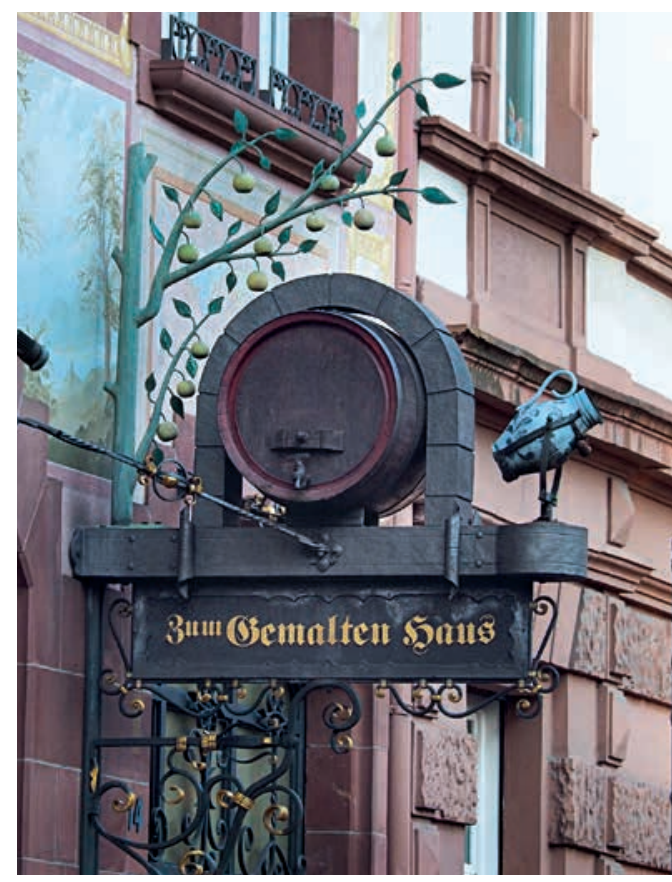

Abb. 1: Aushänger mit Bembel einer Apfelweinkneipe in Frankfurt-Sachsenhausen. (Foto: M. ЈАСОВI) 
konsum im Rhein-Main-Gebiet liegt bei 12 Litern pro Person pro Jahr.

Nachgewiesen wurde das Stöffche, wie der Ebbelwoi hierzulande auch liebevoll genannt wird, in Frankfurt zum ersten Mal 1600. Spätestens 1817 dann hat das „Frankfurter Nationalgetränk“ weltberühmten Status erlangt. Frankfurt lieferte damals in die meisten europäischen Königshäuser und selbst der türkische Sultan trank seinerzeit Ebbelwoi.

Während sich zu dieser Zeit in den Weinregionen der deutschen Landen Strauß- oder Besenwirtschaften ausbildeten, etablierten sich in Frankfurt bald sogenannte „Heckenwirtschaften". Später entwickelten sich aus diesen feste Gaststätten. Ihr Erkennungszeichen: ein Apfel, umgeben von einem Fichtenkranz. Diese Tradition besteht bis heute. Sehnt es eine*n nach Stöffche, suche man nach einem Fichtekranz über der Tür.
Mittlerweile allerdings prangt statt des Apfels meist ein Bembel in dessen Mitte. Verschiedene Theorien ranken sich im Wettstreit um die Herkunft dieser Bezeichnung für das bauchige, hellgraue Gefäß mit blauen Verzierungen, das aus Ton im Ofen gebrannt wird. Die als am plausibelsten gehandelte - und intellektuell anspruchsvollste Theorie führt die Bezeichnung auf den lateinischen Begriff pampinus zurück, was für einen jungen Trieb eines Weinstocks oder auch Weinlaub steht. Als Pampel wurden im 17. Jahrhundert Weingefäße bezeichnet.

Der Aufstieg des Ebbelwois hat übrigens auch mit einer klimatischen Veränderung zu tun. Eine sogenannte „Kleine Eiszeit“ um den Jahrhundertwechsel vom 16. zum 17. Jahrhundert führte zu einer Abkühlung des hiesigen Klimas, die es den bis dato hochproduktiven Weinhängen rund um Frankfurt schwerer machte. Der Apfel indessen bewährte sich bei den kühleren Temperaturen, sodass er von da an vermehrt gepflanzt wurde.

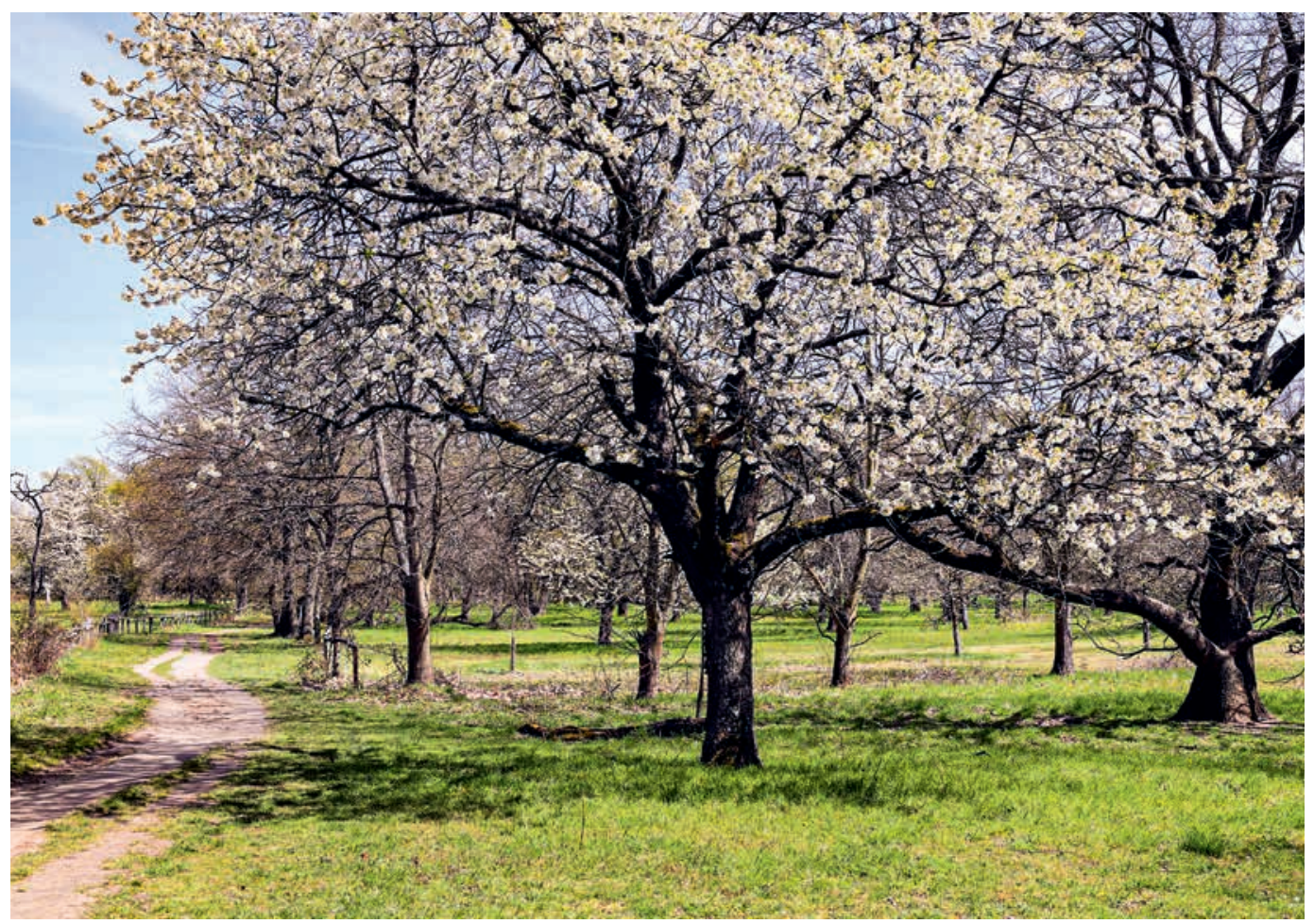

Abb. 2: Alte Streuobstwiese im Frankfurter Naturschutzgebiet „Schwanheimer Düne“. (Foto: H. Steinecke) 


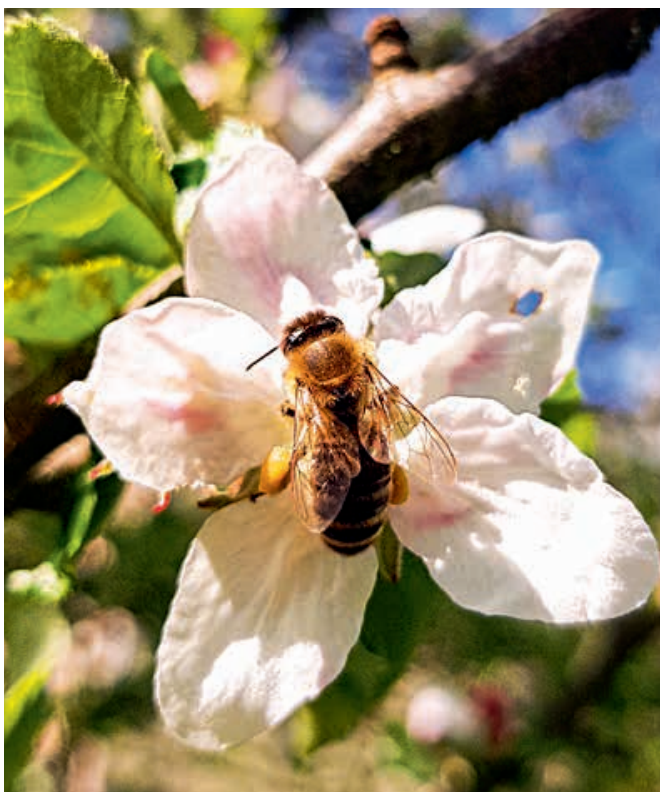

Abb. 3: Honigbiene während ihrer Bestäubungsarbeit an einer Apfelblüte (Foto: T. Collet)

Das endgültige Aus für die Frankfurter Weinkultur und den Durchbruch des Apfelweins beschied dann aber ein ziemlich kleiner Organismus: Ende des 19. Jahrhunderts vernichtete die aus Nordamerika eingeschleppte Reblaus (Daktulosphaira vitifoliae) die Mehrheit der lokalen
Weinstöcke. Die Pflanzenlaus aus der Familie der Zwergläuse ist bis heute eine der bedeutendsten Schädlinge im Weinbau.

Rund um Frankfurt prägen seit dieser Zeit Apfelbäume die Hänge. Streuobstwiesen zählen heute zu einem der bedeutendsten Habitate in der Region, die nicht nur ökologisch, sondern auch kulturell besonders wertvoll sind - von der Bedeutung fürs Stöffche nicht zu reden!

\section{Das Stöffche: vom eigentlichen Ursprung}

Was aber steckt denn nun drin im Stöffche? Ganz klar und einfach zu beantworten: Äpfel. Viel mehr braucht es nicht, um den Ebbelwoi herzustellen, noch ein bisschen Hefe und ein wenig Zucker, für die Produktion des Alkohols. Und dann kann die Herstellung auch schon beginnen.

Aber davor steht etwas ganz Besonderes: die Bestäubung der Apfelblüten. Denn ohne fremde Hilfe durch fleißige Insekten, die den Pollen von Blüte zu Blüte tragen, bildet der Apfelbaum keine guten Früchte aus, und auch deutlich weniger.

$\mathrm{Zu}$ den die Apfelblüten bestäubenden Sechsbeinern zählen neben der bekannten Honigbiene (Apis mellifera) auch zahlreiche Wildbienenarten,

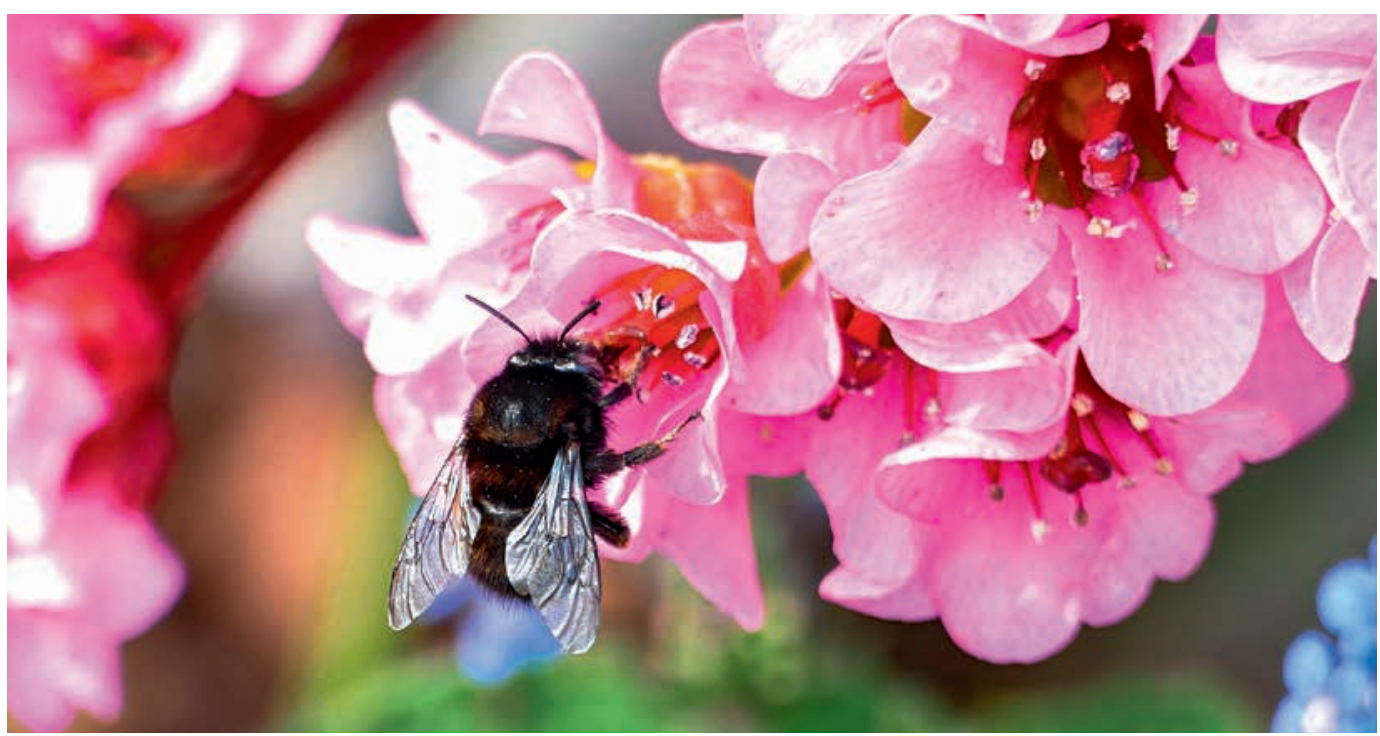

Abb. 4: Wildbienen tragen entscheidend zur Bestäubung von Wild- und Kulturpflanzen bei, hier eine Gemeine Pelzbiene (Anthophora plumipes). (Foto: H. STEINECKe) 
darunter Mauerbienen, Sandbienen, Furchenbienen und Schmalbienen. Auch die Wildbiene mit dem wohlklingenden Namen „Frühlings-Pelzbiene" (Anthophora plumipes) tummelt sich unter den fliegenden Pollenträgern. Ebenso pelzig, nur etwas größer, sind auch zahlreiche Hummeln an der Produktion des Grundstoffs des Ebbelwois intensiv beteiligt: Gartenhummel, Ackerhummel, Erdhummel, Baumhummel, Steinhummel, Kuckuckshummel, Veränderliche Hummel. Sie alle bestäuben die Blüten schon ab einer Temperatur von ca. 8 Grad Celsius und sorgen so schon für die nächste Apfelernte, wenn es der Honigbiene noch zu kalt ist.

Dennoch steht die Honigbiene ganz besonders hoch im Kurs als Bestäuberin für Äpfel. Zwar beginnen sie erst bei höheren Temperaturen, bei rund $15^{\circ} \mathrm{C}$, ihren Stock zu verlassen und auf Nahrungssuche zu gehen. Allerdings erhöht sich durch ihre Bestäubung die Fruchtbildung im Durchschnitt um das Zweieinhalbfache. Der eigentliche „Stöffche-Produzent" ist also die Honigbiene.

Das Stöffche: nur möglich durch eine „Ökosystemleistung". Dass die Insekten bei ihrer Nahrungssuche so ganz nebenbei die Blüten bestäuben, nennt man in der Wissenschaft „Ökosystemleistung “, also eine Leistung der Natur, die sie für den Menschen erbringt - und ohne die wir nicht existieren könnten. Denn die Grundlage dessen, was unser tägliches Überleben sichert - etwa sauberes Wasser, reine Luft, fruchtbare Böden, Nahrung und Bau- und Brennstoffe - sind allesamt Leistungen der Natur, die wir ganz selbstverständlich nutzen.

Die Bestäubungsleistung von Insekten gehört auch dazu. Rund drei Viertel unserer Arten und Sorten vonNahrungspflanzen hängen direkt oder indirekt von der Bestäubung durch Tiere ab. Wie auch bei unserem Apfel sorgen erst sie für die richtige Qualität und Menge. Selbiges gilt für Birnen, Kirschen, Pflaumen, Heidelbeeren und auch Gurken, die zu mehr als 50 Prozent von Tierbestäubung abhängen. Bei den prominenten Beispielen beliebter tropischer Früchte, wie Kiwis, Melonen und Kakao, sind es sogar 100

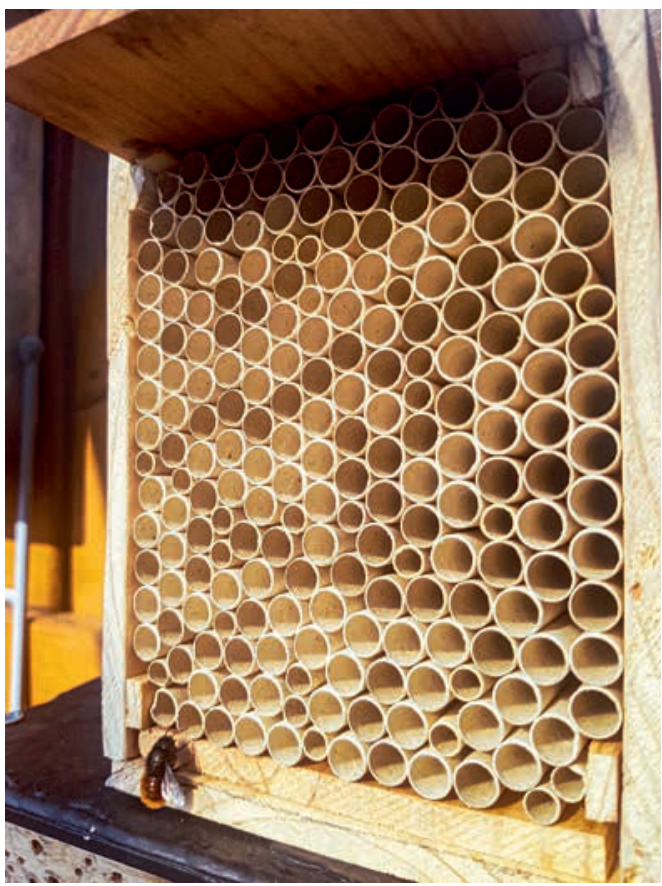

Abb. 5: Beispiel für ein einfaches Insektenhotel, mit dem wir auch in unseren Gärten für manche Arten die Möglichkeit zur Anlage von Brutkammern bieten. Sehr häufige Nutzer von Insektenhotels sind Gehörnte Mauerbienen (Osmia cornuta). (Foto: T. Collet)

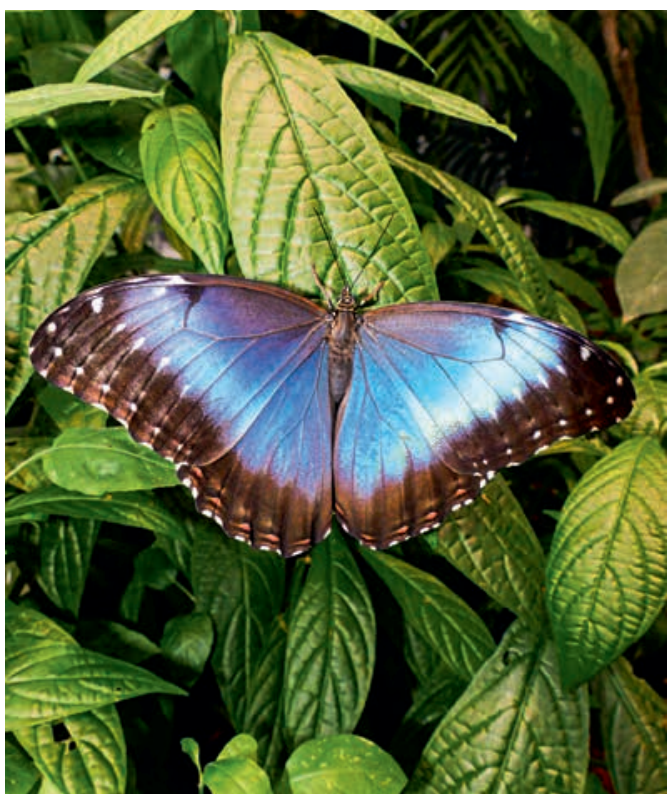

Abb. 6: Himmels- oder Morphofalter (Morpho peleides), eine der auffälligsten Arten im Blüten- und Schmetterlingshaus. (Foto: H. STeinecke) 


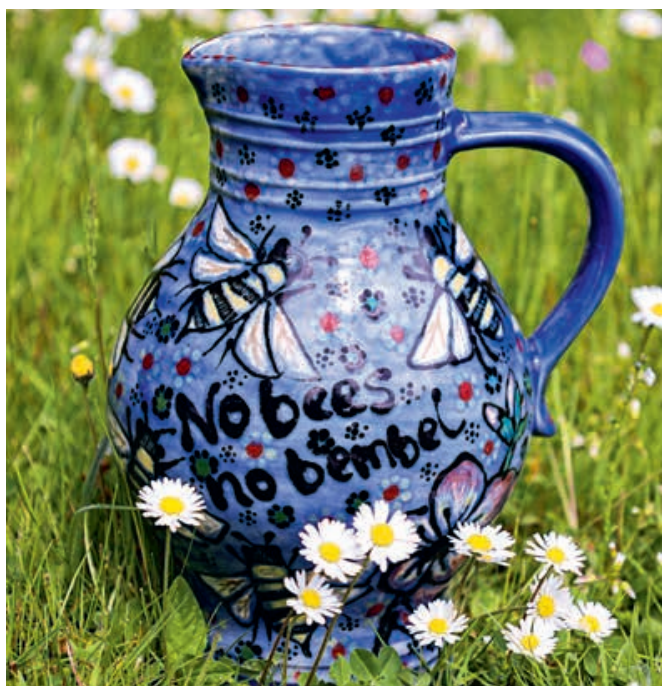

Abb. 7: Der Bembel zum Leitspruch. (Bemalt von und Foto: H. Steinecke)

Prozent. Letztere werden übrigens durch Mücken bestäubt - aber das ist ein anderes Kapitel.

Dass Bestäubung - wohlgemerkt als kostenlose Leistung der Natur - damit auch zu einem bedeutenden Wirtschaftsfaktor wird, erschließt sich sofort. Der morgendliche Kaffee und der Obstsalat zum Frühstück, am Nachmittag dazu das Stück Schokolade und am Abend der Gurkensalat zum Brot - nichts, dass wir missen möchten und vieles, das sich kulturell gar nicht wegdenken lässt. Was sind Ökosystemleistungen also anderes als Kulturstifter? Ein echtes Kaffeekränzchen ohne Schwarzwälder Kirschtorte? - Undenkbar! Oder eben: Ein Stelldichein unterm Fichtekranz ohne Ebbelwoi?

Was also, wenn die Leistungen von Bienen, Hummeln und anderen Insekten plötzlich wegfielen? Das Angebot in unseren Supermärkten wäre ziemlich mager. Könnten wir Bestäubungsleistungen womöglich ersetzen und was würde uns das kosten? Experimente mit dieser Fragestellung - eher unfreiwilliger Art - musste China in den vergangenen Jahren durchführen. Das dortige Bienensterben, verursacht durch massiven Pestizideinsatz, und die gleichzeitige Verwendung von Sorten, die eine zweite Sorte zur Kreuzbestäubung benötigen, führten zu einem drastischen Einbruch der Obstproduktion.
Seitdem werden die Obstbäume von Hand bestäubt, was wahrlich eine große Fleißarbeit ist. Während ein Bienenvolk beispielsweise pro Tag rund 300 Millionen Apfelblüten bestäuben kann, braucht man für dieselbe Produktivität rund 1500 Menschen. Die dauerhaften Kosten für diesen Ersatz natürlicher Leistungen durch menschliche (oder technische) Arbeit mag man sich erst gar nicht ausrechnen. Zum Glück erholen sich die Bienenvölker in China langsam wieder.

\section{Das Stöffche: Ein Leitthema für die Bestäuber}

Unser Ebbelwoi braucht also die Biene und damit die Biene und alle anderen bestäubenden Insekten unseren Schutz. Dazu zählt in erster Linie, dass den Insekten ausreichend Nahrung zur Verfügung steht. Geeignete Nektar- und Futterpflanzen finden sich in Feld und Flur jedoch leider nur noch selten. Vielerorts ist die Landschaft ausgeräumt, artenreiche Wiesen, abwechslungsreiche Felder und Blühgehölze werden seltener. Hinzu kommen schädliche Pestizide, die sich negativ auf das Nervensystem der Insekten auswirken.

Um auf diese Problematik aufmerksam zu machen und darauf, was man dagegen tun kann, haben wir in 2021 ein neues - und gleichzeitig das erste - Leitthema für den Palmengarten und den Botanischen Garten ausgerufen: Blüten- und Bestäuberökologie.

Herzstück und Ausgangspunkt des Leitthemas ist das neue Blüten- und Schmetterlingshaus, das wir in 2021 eröffnen. In einem Teil des 600 Quadratmeter großen Gewächshauses flattern farbenfrohe tropische Schmetterlinge um die Wette, um Klein und Groß zu begeistern. Dabei kann man Bananen-, Monarch- und Morphofalter im Puppenkasten sogar beim Schlüpfen zuschauen.

Die exotischen Falter sind damit gleichzeitig die sympathischen Botschafter für das Thema Bestäubung. Denn auch die Schmetterlinge tropische wie einheimische - gehören zu den bestäubenden Insekten und damit zu den Erbringern dieser Ökosystemleistung. So ist der Übergang 
leicht geschafft in die zugehörige Ausstellung „Abgestaubt! - von Blüten und ihren Bestäubern “im benachbarten Kalthaus, die die große Welt der Insekten unter die Lupe nimmt. Sie zeigt ihre Vielfalt, ihre Form- und Farbenpracht und schafft damit Faszination für die Tiere, die nahezu jeden Lebensraum unserer Erde besiedeln und für uns Menschen von enormem Nutzen sind.

Welchen aktuellen Bedrohungen die Insekten gegenüberstehen und wie wir gegenwirken können, auch davon erzählt die Ausstellung. In den letzten Jahrzehnten hat die Anzahl der Fluginsekten in Deutschland um über 70 Prozent abgenommen. Der Klimawandel, die Intensivierung der Landwirtschaft, Bebauung und Versiegelung von Lebensräumen - all dies zerstört die Lebensgrundlage der Insekten. Indem wir das eigene Konsumverhalten überdenken, ohne Insektizide und Pestizide gärtnern, eine artenreiche Blühwiese statt eines Englischen Rasens anlegen - all das bestimmt auch, ob sich Bienen, Hummeln und Co. weiter für uns und die Ökosysteme einsetzen können.

Das Blüten- und Schmetterlingshaus wird damit zum Ausgangspunkt für Erkundungen in Palmengarten und Botanischen Garten, um Insekten zu beobachten, konkrete Ideen für die Anlage von insektenfreundlichen Beeten zu erhalten, oder auch für Nisthilfen. Ein spezielles Icon, das auf die Ausstellung „Abgestaubt! - von Blüten und ihren Besuchern" hinweist, zeigt den Weg durch die Gärten.

\section{Das Stöffche: Damit es Kulturgut bleibt!}

Und damit zurück zum Stöffche. Um das Frankfurter Nationalgetränk zu bewahren, und damit die Erosion eines Stücks lokaler Geschichte und Identität, gilt es, die Bienen zu schützen und zu fördern. „No Bees, no Bembel“ könnte zum Leitsatz der Hessischen Apfelweinkultur werden stellvertretend für mannigfaltige weitere unserer Kulturgüter, die auf den Leistungen der Natur beruhen.

Ganz in diesem Sinne hat der Verein Apfelwein Centrum Hessen 2020 einen Antrag auf
Aufnahme der hessischen Apfelweinkultur in das Bundesweite Verzeichnis des Immateriellen Kulturerbes der Deutschen UNESCO-Kommission gestellt. Der Apfelwein sei ein zentraler Bestandteil der hessischen Lebensart - ist er auch!

Fehlt für die Zukunft nur noch, die Leistungen der Bestäuber angemessen zu würdigen. Am besten machen wir das, indem wir gut auf sie achtgeben. Wie Sie das machen, erfahren Sie bei uns im Palmengarten und Botanischen Garten.

\section{Internetseiten}

https://www.frankfurt-live.com/frankfurter-gold-was-jederfrankfurter-und-eingeplackte-wissen-sollte-124101.html

https://www.bienenwanderung.de/article/apfel

https://www.bembel-aus-frankfurt.de/historie/

Bestäubung spielt entscheidende Rolle für die KakaoProduktion | Wissen \& Umwelt | DW | 25.05.2010 a-5605504\#: : :text=Wissen\%20\%26\%20Umwelt-,Bestäubung $\% 20$ spielt $\% 20$ entscheidende $\% 20$ Rolle $\% 20$ für $\% 20$ die\%20Kakao-Produktion, mehr\%20Bohnen $\% 20$ reifen $\% 20$ am\%20Kakaobaum.\&text=Dabei\%20fanden $\% 20$ sie $\% 20$ heraus $\% 2$ C\%20dass,Ertrag\%20des\%20Baumes\%20zu $\% 20$ verdoppeln.

https://www.bee-careful.com/de/initiative/menschlichebienen-china/

IPBES (2016): Summary for policymakers of the assessment report of the Intergovernmental Science-Policy Platform on Biodiversity and Ecosystem Services on pollinators, pollination and food production. S. G. Potts, V. L. Imperatriz-Fonseca, H. T. Ngo, J. C. Biesmeijer, T. D. Breeze, L. V. Dicks, L. A. Garibaldi, R. Hill, J. Settele, A. J. Vanbergen, M. A. Aizen, S. A. Cunningham, C. Eardley, B. M. Freitas, N. Gallai, P. G. Kevan, A. Kovács-Hostyánszki, P. K. Kwapong, J. Li, X. Li, D. J. Martins, G. Nates-Parra, J. S. Pettis, R. Rader, and B. F. Viana (Hrsg.). Secretariat of the Intergovernmental Science-Policy Platform on Biodiversity and Ecosystem Services. - Bonn.

\section{Anschrift der Autorin und des Autors}

Dr. Katja Heubach, Palmengarte Frankfurt, Siesmayerstraße 61, 60323 Frankfurt, E-Mail: katja.heubach@stadt-frankfurt.de; http://orcid.org/0000-0002-4298-7750

Torsten Collet, Palmengarten Frankfurt, Siesmayerstraße 61, 60323 Frankfurt, E-Mail: torsten.collet@stadt-frankfurt.de 